\title{
Cognitive task performance under (combined) conditions of a metabolic and sensory stressor
}

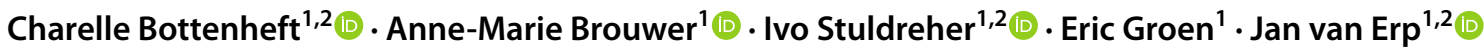

Received: 14 May 2020 / Accepted: 27 September 2020 / Published online: 12 October 2020

(c) The Author(s) 2020

\begin{abstract}
Effects of stressors on cognitive task performance have primarily been studied in isolation, and little is known about the combined effects of two or more stressors. This study examined how a metabolic stressor (skipping breakfast) and a sensory stressor (noise) affect cognitive task performance in isolation and combined. In addition to performance, we collected physiological and subjective data to get insight in the underlying mechanisms. Twenty participants came to the lab twice, once after skipping breakfast, and once after a standardized breakfast. They performed runs of the 2-back task and the International Shopping List Task, which were alternately presented with and without noise. During the 2-back task, electrocardiography (ECG), electrodermal activity (EDA), and electroencephalography (EEG) were recorded. Subjective ratings on effort and stress were also collected. No interaction effects between the two stressors on cognitive performance were found. Skipping breakfast did not cause hypoglycemia, but resulted in subjective discomfort and a lower state of arousal (as indicated by lower heart rate and EDA). These may underly the trend for more missed responses on the 2-back task after breakfast skipping. Noise appeared to generate arousal and increased attention (reflected in higher EDA and P300) in accordance with higher experienced load and stress. This is consistent with less missed 2-back responses in noise conditions. The results indicate that individuals spent extra effort to maintain task performance in the presence of noise. We propose to use a model that, besides additional effort, takes the effect of stressors on performance into account.
\end{abstract}

Keywords Cognition $\cdot$ Workload $\cdot$ Noise $\cdot$ Hypoglycemia $\cdot$ Physiology

\section{Introduction}

In many professions, individuals have to perform cognitive tasks under various physical or mental stressors, or a combination of these. One can think of military personnel, workers in the industrial production sector, or police officers working on challenging tasks while being exposed to different stressors, like noise, psychological stress, thermal heat, or food deprivation during their work. The effect of these stressors on cognitive performance is often negative, but sometimes also positive (Paulus et al. 2009). A systematic review by Martin et al. (2019) indicates that there is a

Charelle Bottenheft

charelle.bottenheft@tno.nl

1 Netherlands Organisation for Applied Scientific Research (TNO), Human Factors, Soesterberg, The Netherlands

2 Human Media Interaction, Computer Science, University of Twente, Enschede, The Netherlands growing interest in the impact of environmental stressors on cognitive performance.

Effects of environmental stressors on performance have usually been studied in isolation (for a review, see Adolphus et al. 2016; Szalma and Hancock 2011), and it has been argued that more research should focus on the effects of combined stressors (Tipton 2012; Lloyd and Havenith 2016). There are only a few studies that investigated the effects of multiple stressors on human performance. For example, Lieberman et al. (2006) found cognitive decrements when participants were exposed to a military relevant multi-stressor environment including food deprivation, sleep loss, and physical activity lasting for $84 \mathrm{~h}$. The cognitive tasks involved simple attention tasks and a working memory task. However, they did not compare the effects of the individual stressors to the multi-stressor environment, so that no interaction effects could be investigated. Other studies did examine interaction effects of two environmental stressors, but only on physical performance (Lloyd et al. 2015, 2016). They examined either cold or heat load in combination with 
hypoxia (high altitude, $4100 \mathrm{~m}$ ) and compared this to stressors in isolation. They found reduced physical performance for both combinations, but the type of interaction differed between combined hypoxia and cold (additive) and hypoxia and heat (antagonistic). In theory, there are three different types of interactions possible: (1) additive, meaning the sum of the individual effects of each stressor, (2) synergistic, meaning a larger combined effect than the sum of the individual stressors, and (3) antagonistic, meaning a smaller combined effect than the sum of the individual stressors (Lloyd and Havenith 2016). Although their studies focused on physical performance, the results are interesting, because they suggest that the interaction type depends on whether or not the two stressors share a common underlying mechanism (Lloyd et al. 2015). Combining stressors that share mechanisms may result in synergistic or antagonistic interactions, while combining stressors with independent mechanisms results in additive effects. Interactions are also influenced by the magnitude of the effect of individual stressors. Mild stressors can add up, while the greater the impact of stressors, the greater the trend for one stressor to mask the effect of the other stressor (Lloyd and Havenith 2016).

The objective of this study is to explore the effects of a combination of two stressors on cognitive performance. We selected two stressors based on the assumption that they impact the human body through different mechanisms: a sensory stressor and a metabolic stressor. The former comprised noise bursts during task execution, and the latter comprised skipping breakfast before the task. Although many different combinations of stressors are possible, the selected stressors have operational relevance. Noise bursts are commonly present in professional environments. For example, workers in the industrial production sector or military personnel often operates in noisy environments, involving both exposures to long-duration noise and acute repetitive impulse noises (Yong and Wang 2015). Noise bursts generally impair cognitive processes, such as attention and working memory (Matthews et al. 2000). This has been attributed to a degradation of the information that is held in working memory through distraction by the task-irrelevant noise (Hockey 1986 in Szalma and Hancock 2011). The other stressor, skipping breakfast, is also relevant for professionals, such as military pilots who sometimes have to skip meals when missions are extended or workers in the industrial production sector who forget or skip a meal during a long work day. Two review studies have shown that breakfast consumption has an acute positive effect on cognition in healthy participants, specifically on memory, attention, and executive functioning (Adolphus et al. 2016; Galioto and Spitznagel 2016). Skipping breakfast can lead to a hypoglycemic situation (Galioto and Spitznagel 2016), leaving insufficient energy for the brain to function optimally. Memory, one of the main aspects of cognition, is associated with medial temporal and mainly the hippocampal regions of the brain, which are known to be vulnerable to hypoglycemia (Benton and Parker 1998). Also, sensations of hunger and stress may cause low motivation to perform a cognitive task (Hoyland et al. 2009).

In addition to examining the effects of the combination of stressors on cognitive task performance, we collected subjective and physiological data to get insight into underlying mechanisms. This arises from the idea that physiological states influence cognition (Critchley and Garfinkel 2018). Combining performance, physiological, and subjective measures of mental effort and workload contributes to a more complete understanding of the individual's state as compared to performance alone. For instance, task performance may not be affected by a stressor, but this does not necessarily mean that the stressor does not have an effectindividuals may spend more effort to maintain performance level. Higher mental effort, or workload, is related to a decrease of the parasympathetic activity of the autonomic nervous system, which can be viewed as the "rest and recovery" system and an increase in sympathetic activity, or the "fight or flight" system (Mulder and Mulder 1987). Also measures of central nervous system activity, for example obtained through the electroencephalogram (EEG), are reflective of higher mental effort (Brouwer et al. 2012). We chose a broad range of physiological measures that reflect changes in these central and autonomic nervous systems, which may be expected to underly processes that relate to (maintaining) cognitive performance, such as attention and arousal. These measures are explained in more detail in the method section.

\section{Method}

\subsection{Participants}

Twenty-one participants took part in this study, of which 20 completed the whole study. They were recruited through the TNO participant pool or acquainted with one of the test leaders. An approval for this study was granted by the TNO Internal Review Board (reference number: 2019-011). All participants gave written informed consent. Participants were aged between 18 and 55 years (mean age 39.9 years) and 11 of them were male.

\subsection{Materials}

\subsubsection{Cognitive task performance}

For each of two test days, participants performed 16 times a 2-min 2-back task (Kirchner 1958). This task required participants to watch a sequence of letters on a computer monitor. For each letter, participants pressed one of two different 
buttons, depending on whether the letter was a target or a non-target. A letter was a target when it was the same as the letter presented two positions earlier. $33 \%$ of the letters were targets. Each letter was presented for $500 \mathrm{~ms}$ followed by a $1500 \mathrm{~ms}$ inter-stimulus interval. Thus, 62 letters were presented in each 2-min run of which the last 60 were used to analyze performance. Task performance was measured by means of reaction time after each letter, the number of correct responses (computed only for the responses where a button was pressed), and the number of missed responses (i.e., responses where no button was pressed). A 2-back version was chosen, because many participants find the 3-back task too difficult, get overwhelmed, and tend to give up (Izzetoglu et al. 2007; Ayaz et al. 2007).

A Dutch version of the International Shopping List Task (ISLT; Thompson et al. 2011) was used to measure verbal list learning and memory. The ISLT challenged participants with immediate and delayed recall of 12 words in random order (free recall). Computer software presented the words to the test leader who immediately read these words to the participant. Words were presented to the test leader at a rate of one word per $2 \mathrm{~s}$. After the 12 words, the participants were asked to recall as many words as they could remember (immediate recall). The same list, followed by immediate recall, was repeated a total of three times. After approximately $20 \mathrm{~min}$, participants were asked to recall the same 12 words (delayed recall). Task performance consists of total number of immediate recalled words (total recall, range $0-36)$ and the words recalled during the delayed recall trial (range 0-12).

\subsubsection{Subjective questionnaires}

Feelings of hunger were measured with a visual analogue scale (VAS) at the time of arrival on both test days. This VAS scale runs from 0 ('not at all') to 100 ('extremely'). This scale was used as manipulation check for skipping breakfast. After each 2-min run, the participants rated their experienced stress on a VAS, and their subjective mental effort on the Rating Scale of Mental Effort (RSME) (Zijlstra and van Doorn 1985). The RSME scale ranges from 0 to 150 , with higher values reflecting higher workload. It has nine descriptors along the axis, e.g., 'not effortful' at value 2 and 'rather effortful' at value 58.

\subsubsection{Physiological measurements}

During the 2-back task, electrocardiography (ECG), electrodermal activity (EDA), and electroencephalography (EEG) were recorded. Both heart rate, derived from ECG, and EDA increase with working memory load; heart rate variability, also derived from ECG, decreases with working memory load (Brouwer et al. 2014). EDA is a robust indicator of arousal
(Mendes 2009). The individual's state is also reflected by changes in electrical brain activity (EEG). The P300 component of an event-related potential (ERP) reflects attentional and working memory processes (Polich 2007). A higher amplitude indicates attention to the evoking events (Wild-Wall et al. 2011; Evans et al. 2011). The alpha activity (power in the EEG 8-12 $\mathrm{Hz}$ band) has been associated with active inhibitory mechanisms (Brouwer et al. 2009), idling (Pfurtscheller et al. 1996), and default mode brain activity (Laufs et al. 2003; Jann et al. 2009). This suggests that alpha would reflect different levels of workload, with high alpha being inversely related to levels of workload (Fink et al. 2005; Brouwer et al. 2012). Another EEG frequency band related to workload processes is theta activity (power in the EEG $4-8 \mathrm{~Hz}$ band) and can be an indication of increasing task requirements (Jensen and Tesche 2002).

ECG, EDA, and EEG were all recorded at $1024 \mathrm{~Hz}$ using an ActiveTwo Mk II system (Biosemi, Amsterdam, The Netherlands), such that variables are stored together with event codes of experimental events in one, synchronized data matrix. For ECG, two active gelled $\mathrm{Ag}-\mathrm{AgCl}$ electrodes were placed at the right collarbone and lowest floating left rib. For EDA, two passive gelled Nihon Kohden electrodes were placed on the ventral side of the distal phalanges of the left middle and index finger on the hand that was not used for pressing the response keys. EEG was recorded with 32 active $\mathrm{Ag}-\mathrm{AgCl}$ electrodes, placed on the scalp in the standard 10-20 arrangement (Jasper 1958), together with a common mode sense active electrode and driven right leg passive electrode for referencing. These two electrodes form a feedback loop that replace the ground electrode common in other EEG systems, but have the same basic function of being an electrical reference for the other electrodes. The electrode impedance threshold was set at 20 $\mathrm{kOhm}$.

\subsubsection{Glucose and cortisol}

Prior to cognitive testing, the level of blood glucose was assessed using a finger prick blood sample. The finger prick was done approximately $180 \mathrm{~min}$ after having breakfast (or not). In addition to the subjective hunger ratings, this measure was used as manipulation check for skipping breakfast. The Bayer Contour TS system was used for determining the amount of glucose in blood. Salivary cortisol was determined on awakening as well as 30 and 45 min after awakening, and in the laboratory on arrival and at the end of the experiment. The cortisol analysis will not be discussed in this article.

\subsection{Design}

The design was a within-subjects $2 \times 2$ design: noise (noise bursts, no-noise bursts) $\times$ breakfast (skipping breakfast, standardized breakfast). 


\subsubsection{Breakfast}

Participants came to the lab twice, once after skipping breakfast, and once after eating a standardized breakfast. The order of (skipping) breakfast was counterbalanced. The 2 test days were at least 1 week apart. The condition 'skipping breakfast' was operationalized as fasting from $8 \mathrm{pm}$ the night before the test day. Drinking water was allowed. The standardized breakfast consisted of $150 \mathrm{~g}$ of plain quark, $25 \mathrm{~g}$ of roasted nut mix, a pinch of cinnamon, and at least one glass of water (fat: $28 \mathrm{~g}$, protein: $16.8 \mathrm{~g}$, total carbohydrates: $6.9 \mathrm{~g}$, and mono- and disaccharides carbohydrates: $4.9 \mathrm{~g}$ ). Participants were instructed to eat their breakfast at 7:30 am. This was about $180 \mathrm{~min}$ before the cognitive tasks were completed. The reason for this is that positive effects of breakfast on cognitive performance are most often found at about $180 \mathrm{~min}$ after breakfast consumption (Adolphus et al. 2016). Participants in the breakfast condition were not allowed to eat anything else besides the standardized breakfast from $8 \mathrm{pm}$ the night before the test day. Drinking water was allowed.

\subsubsection{Noise bursts}

During each test day, participants performed the experiment in two blocks. Each block contained immediate- and delayed recall of the ISLT and eight runs of the 2-back task. The immediate recall task was performed once with noise bursts and once without noise bursts. The order of (no-)noise during the ISLT was counterbalanced. 2-min runs were alternately presented with and without white noise bursts played through speakers. Thus, eight runs were presented with noise bursts and eight runs without noise bursts (see Fig. 1). This order was also counterbalanced. In the noise run, $500 \mathrm{~ms}$ white noise burst $(85 \mathrm{~dB}$ at random center frequencies at
$1000 \mathrm{~Hz}, 2000 \mathrm{~Hz}$ and $4000 \mathrm{~Hz}$ ) were used as noise stimuli. They were presented together with two-thirds of the letters. Participants were informed in advance whether a run was with or without noise bursts.

\subsection{Procedures}

Participants were instructed to come to the lab twice, always on a weekday at 9 a.m. to avoid confounding circadian influences. Participants received a cortisol saliva set consisting of two pockets with each five saliva samples and a standardized breakfast at home, which they had to eat at 7:30 am. They were instructed to collect three saliva samples on both test days. After entering the lab, participants were instructed about the procedures. Participants started the test day with a baseline saliva sample. Next, they performed a 10-min physical exercise on an ergometer (9:20 am), with the assumption that such physical activity would lower the blood glucose level even more after skipping breakfast. This exercise was done on both test days. During this exercise, the participant wore a heart rate band to control for physical exhaustion (heart rate higher than 220 beats per minute minus age). After a rest period, the EDA, ECG, and EEG sensors were attached. Next, all participants practiced the 2-back task at least four times and until the participants felt comfortable that they mastered the task. After this practice, a finger prick blood sample was taken (10:10 am), followed by a 5-min rest period. Then, the first ISLT was conducted, followed by eight times the 2-back task and the delayed recall of the ISLT (10:20 am). This was repeated twice. After performing each 2-min run of the 2-back task, the participants rated subjective mental effort on the RSME and stress on a visual analogue scale. At the end of the test day, the last saliva sample for cortisol measures was taken.

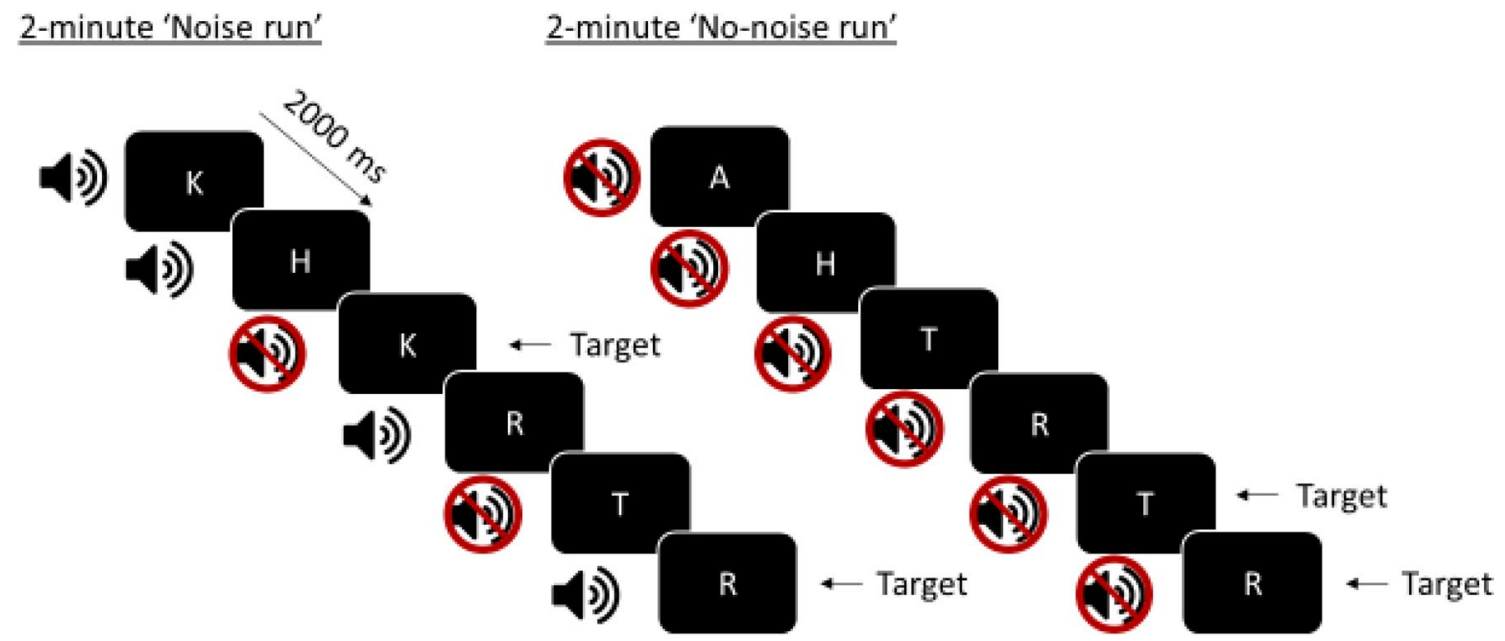

Fig. 1 Schematic depiction of the sequence of targets and non-targets in the 2-back task in a 'noise' and 'no-noise' run 


\subsection{Data analysis}

\subsubsection{Pre-processing}

Data pre-processing was done with MATLAB 2019a software (Mathworks, Natick, MA, USA).

ECG data were down sampled to $256 \mathrm{~Hz}$ and high-pass filtered at $0.5 \mathrm{~Hz}$. R-peaks in the ECG signal were detected following Pan and Tompkins (1985), resulting in a semitime-series of consecutive IBIs. We computed the mean inter-beat interval (IBI), i.e., the interval between consecutive R-peaks of the ECG, and the root mean squared of successive IBI differences (RMSSD) metric of heart rate variability (HRV) for each 2-min 2-back run. Outliers were removed in an iterative way with four repetitions. In each repetition, values more than four standard deviations from the mean were replaced with not-a-number $(\mathrm{NaN})$ values.

EDA was downsampled to $64 \mathrm{~Hz}$. The fast phasic component was separated using Continuous Decomposition Analysis as implemented in the Ledalab Toolbox for MATLAB (Benedek and Kaernback 2010). The number of different sets of initial values to be considered in the optimization was kept at the default value of two. Next, variables of the EDA were obtained from the Ledalab analysis. In each 2-min epoch, the mean phasic peak amplitude and number of phasic peaks were obtained. The peak amplitude threshold was set to $0.05 \mu \mathrm{S}$, and peaks with lower amplitudes were discarded. Outliers were removed in an iterative way with four repetitions. In each repetition, values more than four standard deviations from the mean were replaced with not-a-number $(\mathrm{NaN})$ values.

EEG was processed using EEGLAB v14.1.2 for MATLAB (Delorme and Makeig 2004). EEG was first downsampled to $256 \mathrm{~Hz}$ and band-pass filtered between 1-30 Hz. Bad channels were removed based on their statistical properties. Channels with a standard deviation exceeding $50 \mu \mathrm{V}$ were removed. Then, within kept channels, samples exceeding $\pm 150 \mu \mathrm{V}$, as well as samples within $100 \mathrm{~ms}$, were also removed. After interpolating bad channels, channels were rereferenced to the average channel values. Logistic infomax ICA (Bell and Sejnowski 1995) was performed to localize independent components. The Multiple Artifact Rejection Algorithm (MARA) (Winkler et al. 2011) was executed to classify artifactual independent components, i.e., components not reflecting sources of neural activity, but ocular or muscle-related artifacts. MARA is a supervised learning algorithm that learns from expert ratings of 1290 components. It classifies components based on six features from the spatial, spectral, and temporal domain. The artifactual components were removed from the data. EEG were cut in 2-min epochs corresponding to the 162 -back runs. For each epoch, the EEG frequency power spectrum was computed using the EEGLAB 'spectopo' function. The Alpha power at the midline-parietal site (electrode $\mathrm{Pz}$ ) and Theta power at the midline-frontal site $(\mathrm{Fz})$ were computed as the mean power in the $8-12 \mathrm{~Hz}$ and $4-8 \mathrm{~Hz}$ range, respectively. EEG data were then cut into $1100 \mathrm{~ms}$ epochs starting $100 \mathrm{~ms}$ before the onset of letters. Only letters without noise bursts were considered in the analysis (i.e., in no-noise runs all letters were considered and in noise runs only letters without noise). For each 2-min run in each participant, the grandaverage event-related potential (ERP) at $\mathrm{Pz}$ was computed for targets and non-targets. The mean P300 amplitude for targets and non-targets in each 2-min run was computed as the maximum value of the grand-average ERP between 200-400 ms after target and non-target onset, respectively.

\subsubsection{Statistical analysis}

Statistical analyses were performed using SPSS software version 25.0. The dependent variables were tested with two-way repeated-measures analysis of variance (ANOVA). Effects of both two-level independent variables, noise and breakfast, on performance variables and psychophysiological measures were tested. Only for EEG P300 response, an additional within-subjects $2 \times 2$ design was included to analyze the effect of type of letter (target, non-target) and noise (noise bursts, no-noise bursts) on the P300 amplitude. The non-parametric variant (Wilcoxon Matched-Pairs SignedRank test) was used for the subjective ratings. To examine the effect of skipping breakfast on reported feelings of hunger and glucose levels in blood, paired-sample $T$ tests were used. The non-parametric variant (Wilcoxon Matched-Pairs Signed-Rank test) was used for reported feelings of hunger. All statistical tests were performed at a significance level of alpha $=0.05$.

\section{Results}

A total of 21 participants were included in this study at the start. Because one participant did not show up on the second test day, data of 20 participants (10 male and 10 females, mean age 37.1) were analyzed. EDA data from one participant were removed due to what seemed to be excessive movement artifacts.

\subsection{Manipulation check for skipping breakfast}

Reported feelings of hunger and glucose were examined on both test days, with and without breakfast consumption. Values for both test days can be found in Table 1. A Wilcoxon Signed-Rank Test showed that feelings of hunger were significantly higher after skipping breakfast compared to standardized breakfast, $z=-3.82, p<0.001$. A pairedsample $T$ test showed that the level of blood glucose did 
not significantly differ between both test days, $t(18)=-0.31$, $p=0.763$.

Means and standard deviations for each variable in each of the four stressor conditions are listed in Table 2. An overview of the (close to) significant and non-significant results are described and listed in Table 3.

\subsection{Results of cognitive task performance}

Regarding the 2-back task, statistical analysis did not show a main effect of breakfast on reaction time and number of correct responses. A trend was found for number of missed responses on the 2-back task, $F(1,19)=3.95, p=0.061$, with more missed responses after skipping breakfast $(M=1.13 ; \mathrm{SE}=0.50)$ compared to standardized breakfast $(M=0.74 ; \mathrm{SE}=0.41)$. There was no effect of noise on mean reaction time and number of correct responses of the 2-back task. However, noise significantly affected the number of missed responses, with more missed responses in the no-noise burst condition $(M=1.08 ; \mathrm{SE}=0.46) \mathrm{com}$ pared to the noise bursts condition $(M=0.79 ; \mathrm{SE}=0.44)$, $F(1,19)=8.75, p=0.008$. We found no interaction effects of breakfast and noise on any of the 2-back performance variables (mean reaction time, number of correct responses, and number of missed responses).

Regarding the ISLT, there was no main effect for breakfast on total recall or on delayed recall. There was also no effect of noise on total recall. However, noise significantly affected the delayed recall, with less recalled words in the noise bursts condition $(M=9.42 ; \mathrm{SE}=0.43)$ compared to the no-noise burst condition $(M=10.05$; $\mathrm{SE}=0.43), F(1,19)=8.05, p=0.011$. No interaction

Table 1 Reported feeling of hunger and blood glucose examined $(\mathrm{N}=$ number of participants, Mdn= Median, $\mathrm{M}=\mathrm{mean}$, IQR =interquartile range, and $\mathrm{SD}=$ standard deviation) after a standardized breakfast compared to skipping breakfast

\begin{tabular}{|c|c|c|c|c|c|c|}
\hline & \multirow[b]{2}{*}{$\mathrm{N}$} & \multicolumn{2}{|c|}{ Standardized breakfast } & \multicolumn{3}{|c|}{ Skipping breakfast } \\
\hline & & Mdn & IQR & Mdn & IQR & $p$ value \\
\hline \multirow{2}{*}{$\begin{array}{l}\text { Reported feeling of hunger } \\
(0-100)\end{array}$} & 20 & 16.0 & 28.5 & 64.7 & 38.6 & $<0.001$ \\
\hline & $\mathrm{N}$ & M & SD & M & SD & $p$ value \\
\hline Glucose $(\mathrm{mmol} / \mathrm{L})$ & 19 & 5.2 & 0.5 & 5.1 & 0.6 & 0.763 \\
\hline
\end{tabular}

Table 2 Descriptives for each variable in each of the four stressor conditions

\begin{tabular}{|c|c|c|c|c|}
\hline & \multicolumn{2}{|c|}{ Standardized breakfast } & \multicolumn{2}{|c|}{ Skipping breakfast } \\
\hline & Noise bursts & No-noise bursts & Noise bursts & No-noise bursts \\
\hline \multicolumn{5}{|l|}{ 2-back task } \\
\hline Reaction time (ms) & $569 \pm 167$ & $564 \pm 148$ & $574 \pm 167$ & $567 \pm 158$ \\
\hline Number of correct responses & $52.3 \pm 5.2$ & $52.2 \pm 5.3$ & $51.8 \pm 5.3$ & $51.8 \pm 5.2$ \\
\hline Number of missed responses & $0.7 \pm 1.9$ & $0.8 \pm 1.8$ & $0.9 \pm 2.0$ & $1.4 \pm 2.6$ \\
\hline \multicolumn{5}{|l|}{ International Shopping List Task } \\
\hline Total recall trial 1-3 (0-36 words) & $26.3 \pm 4.5$ & $27.0 \pm 3.4$ & $26.1 \pm 5.4$ & $25.9 \pm 4.3$ \\
\hline Delayed recall (0-12 words) & $9.5 \pm 2.1$ & $10.4 \pm 2.5$ & $9.4 \pm 2.5$ & $9.8 \pm 1.9$ \\
\hline \multicolumn{5}{|l|}{ Subjective measures } \\
\hline Reported mental effort $(0-150)$ & $67.8 \pm 29.1$ & $64.4 \pm 39.3$ & $78.3 \pm 37.5$ & $75.8 \pm 38.6$ \\
\hline Reported stress (0-100) & $35.1 \pm 21.9$ & $32.3 \pm 24.1$ & $32.7 \pm 30.1$ & $33.3 \pm 29.3$ \\
\hline \multicolumn{5}{|l|}{ Physiology } \\
\hline ECG-IBI (s) & $0.79 \pm 0.12$ & $0.79 \pm 0.12$ & $0.86 \pm 0.09$ & $0.86 \pm 0.09$ \\
\hline ECG-RMSSD (s) & $0.08 \pm 0.11$ & $0.08 \pm 0.11$ & $0.05 \pm 0.02$ & $0.05 \pm 0.03$ \\
\hline EDA-amplitude phasic $(\mu \mathrm{S})$ & $0.18 \pm 0.10$ & $0.17 \pm 0.09$ & $0.14 \pm 0.07$ & $0.13 \pm 0.06$ \\
\hline EDA-number of peaks & $11.32 \pm 8.25$ & $10.39 \pm 7.85$ & $8.30 \pm 6.39$ & $6.97 \pm 5.78$ \\
\hline EEG-alpha activity $\left(\mu \mathrm{V}^{\wedge} 2 / \mathrm{Hz}\right)$ & $0.69 \pm 0.68$ & $0.73 \pm 0.78$ & $0.86 \pm 0.98$ & $0.87 \pm 1.00$ \\
\hline EEG-theta activity $\left(\mu \mathrm{V}^{\wedge} 2 / \mathrm{Hz}\right)$ & $1.54 \pm 1.35$ & $1.61 \pm 1.51$ & $1.86 \pm 1.55$ & $1.83 \pm 1.57$ \\
\hline EEG-amplitude P300 $(\mu \mathrm{V})$ for target letters & $5.19 \pm 2.10$ & $3.74 \pm 1.99$ & $5.48 \pm 2.31$ & $3.88 \pm 1.62$ \\
\hline EEG-amplitude P300 $(\mu \mathrm{V})$ for non-target letters & $4.16 \pm 1.61$ & $3.15 \pm 1.35$ & $3.97 \pm 1.71$ & $3.12 \pm 1.31$ \\
\hline
\end{tabular}

Values are presented as means \pm standard deviations, except, for subjective measures, the values are presented as median \pm interquartile range 
Table 3 Overview of (close to) significant and non-significant results of stressors

\begin{tabular}{|c|c|c|c|}
\hline & Noise bursts & Breakfast & Noise $\times$ breakfast \\
\hline \multicolumn{4}{|l|}{ 2-back task } \\
\hline Reaction time (ms) & $\mathrm{F}(1,19)=0.77, p=0.392$ & $\mathrm{~F}(1,19)=0.70, p=0.795$ & $\mathrm{~F}(1,19)=0.04, p=0.854$ \\
\hline Number of correct responses & $\mathrm{F}(1,19)=0.12, p=0.734$ & $\mathrm{~F}(1,19)=0.86, p=0.367$ & $\mathrm{~F}(1,19)=0.04, p=0.838$ \\
\hline Number of missed responses & $\begin{array}{l}\mathrm{F}(1,19)=8.75, p=0.008 \\
\text { Lower for noise bursts }\end{array}$ & $\begin{array}{l}\mathrm{F}(1,19)=3.95, p=0.061 \\
\text { (Higher for skipping breakfast) }\end{array}$ & $\mathrm{F}(1,19)=0.62, p=0.440$ \\
\hline \multicolumn{4}{|l|}{ International Shopping List Task } \\
\hline Total recall trial 1-3 (0-36 words) & $\mathrm{F}(1,19)=0.23, p=0.636$ & $\mathrm{~F}(1,19)=1.20, p=0.287$ & $\mathrm{~F}(1,19)=0.48, p=0.498$ \\
\hline Delayed recall (0-12 words) & $\begin{array}{l}\mathrm{F}(1,19)=8.05, p=0.011 \\
\text { Lower for noise bursts }\end{array}$ & $\mathrm{F}(1,19)=1.36, p=0.259$ & $\mathrm{~F}(1,19)=0.27, p=0.607$ \\
\hline \multicolumn{4}{|l|}{ Subjective measures } \\
\hline Reported mental effort $(0-150)$ & $\begin{array}{l}z=-1.83, p=0.067 \\
\underline{\text { (Higher for noise bursts) }}\end{array}$ & $z=-0.75, p=0.455$ & - \\
\hline Reported stress $(0-100)$ & $\begin{array}{l}\mathrm{Z}=-2.61 p=0.009 \\
\text { Higher for noise bursts }\end{array}$ & $z=-0.15, p=0.881$ & - \\
\hline \multicolumn{4}{|l|}{ Physiology } \\
\hline ECG-IBI (s) & $\mathrm{F}(1,19)=2.15, p=0.159$ & $\begin{array}{l}\mathrm{F}(1,19)=7.01, p=0.016 \\
\text { Larger for skipping breakfast }\end{array}$ & $\mathrm{F}(1,19)=0.58, p=0.454$ \\
\hline ECG-RMSSD (s) & $\mathrm{F}(1,19)=0.09, p=0.767$ & $\mathrm{~F}(1,19)=0.96, p=0.339$ & $\mathrm{~F}(1,19)=2.60, p=0.123$ \\
\hline EDA-amplitude phasic $(\mu \mathrm{S})$ & $\begin{array}{l}\underline{\mathrm{F}(1,18)=4.40, p=.050} \\
\underline{\text { Higher for noise bursts }}\end{array}$ & $\begin{array}{l}\mathrm{F}(1,18)=5.81, p=.027 \\
\text { Lower for skipping breakfast }\end{array}$ & $\mathrm{F}(1,18)=0.03, p=.868$ \\
\hline EDA-number of peaks & $\begin{array}{l}\underline{\mathrm{F}(1,18)=15.27} p=0.001 \\
\underline{\text { Higher for noise bursts }}\end{array}$ & $\begin{array}{l}\underline{\mathrm{F}(1,18)=3.99, p=0.061} \\
\text { Lower for skipping breakfast }\end{array}$ & $\mathrm{F}(1,18)=0.35, p=0.564$ \\
\hline EEG-alpha activity $\left(\mu \mathrm{V}^{\wedge} 2 / \mathrm{Hz}\right)$ & $\mathrm{F}(1,19)=2.99, p=0.100$ & $\mathrm{~F}(1,19)=2.47, p=0.133$ & $\mathrm{~F}(1,19)=1.32, p=0.265$ \\
\hline EEG-theta activity $\left(\mu \mathrm{V}^{\wedge} 2 / \mathrm{Hz}\right)$ & $\mathrm{F}(1,19)=0.67, p=0.423$ & $\mathrm{~F}(1,19)=1.55, p=0.229$ & $\mathrm{~F}(1,19)=3.12, p=0.094$ \\
\hline $\begin{array}{l}\text { EEG-amplitude } \mathrm{P} 300(\mu \mathrm{V}) \text { for } \\
\text { target letters }\end{array}$ & $\begin{array}{l}\mathrm{F}(1,19)=49.31, p<0.001 \\
\text { Higher for noise bursts }\end{array}$ & $\mathrm{F}(1,19)=1.42, p=0.249$ & $\mathrm{~F}(1,19)=0.16, p=0.698$ \\
\hline \multirow{2}{*}{$\begin{array}{l}\text { EEG-amplitude } \mathrm{P} 300(\mu \mathrm{V}) \text { for non- } \\
\text { target letters }\end{array}$} & $\begin{array}{l}\text { Higher for noise bursts } \\
\mathrm{F}(1,19)=71.63, p<0.001\end{array}$ & $\mathrm{~F}(1,19)=0.29, p=0.598$ & $\mathrm{~F}(1,19)=0.53, p=0.477$ \\
\hline & Noise & Type of letter & ype of letter \\
\hline $\begin{array}{l}\text { EEG-amplitude P300 }(\mu \mathrm{V}) \text { for } \\
\text { type of letters }\end{array}$ & $\begin{array}{l}1,19)=65.24, p<0.001 \\
\text { gher for noise bursts }\end{array}$ & $\begin{array}{l}\mathrm{F}(1,19)=22.67, p<0.001 \\
\text { Higher for target letters }\end{array}$ & $\begin{array}{l}13.67, p=0.002 \\
\text { sts increased the dif- } \\
\text { ion between target and } \\
\text { et letters }\end{array}$ \\
\hline
\end{tabular}

A significant effect is indicated with a description of the effect and a trend is indicated with a description between brackets. Trends and effects are both underlined.

effects of breakfast and noise were found on total recall or delayed recall.

\subsection{Results of subjective ratings}

Regarding the subjective ratings, no effects of breakfast were found on reported mental effort. A trend was found for noise on reported mental effort, $z=-1.83, p=0.067$, with higher ratings for mental effort during noise bursts ( $\mathrm{Mdn}=68.90, \mathrm{IQR}=33.42)$ compared to no-noise bursts $(\mathrm{Mdn}=63.80, \mathrm{IQR}=39.04)$. There was also an effect of noise on reported stress $(z=-2.61 p=0.009)$, with higher reported stress during noise bursts $(\mathrm{Mdn}=31.90$,
$\mathrm{IQR}=27.45)$ compared to no-noise bursts $(\mathrm{Mdn}=31.32$, $\mathrm{IQR}=27.77$ ).

\subsection{Results of physiological variables}

A significant effect of breakfast was found on the inter-beat interval (IBI) of the ECG signal, $F(1,19)=7.01, p=0.016$, with a higher mean IBI (i.e., lower heart rate) after skipping breakfast $(M=0.86 ; \mathrm{SE}=0.02)$ compared to standardized breakfast $(M=0.79 ; \mathrm{SE}=0.03)$. There was no effect of breakfast on RMSSD (heart rate variability). There were no effects of noise on IBI and RMSSD. Also no interaction effects of breakfast and noise on IBI or on RMSSD were found. 
Regarding EDA, a significant effect of breakfast was found on mean phasic peak amplitude, $F(1,18)=5.81, p=0.027$, and the number of phasic peaks, $F(1,18)=3.99, p=0.061$ (trend). Skipping breakfast decreased the mean phasic peak amplitude $(M=0.138 ; \mathrm{SE}=0.014)$ and the number of phasic peaks $(M=7.63 ; \mathrm{SE}=1.37)$ compared to standardized breakfast (mean phasic peak amplitude: $M=0.171$; $\mathrm{SE}=0.021$, and number of phasic peaks: $M=10.86, \mathrm{SE}=1.84)$. There was also an effect of noise on mean phasic peak amplitude, $F(1,18)=4.40, p=0.050$, and number of phasic peaks, $F(1,18)=15.27, p=0.001$. Increased mean phasic peak amplitude $(M=0.161 ; \mathrm{SE}=0.018)$ and number of phasic peaks $(M=9.81 ; \mathrm{SE}=1.47)$ were found for the noise burst condition compared to the no-noise burst condition (mean phasic peak amplitude: $M=0.148 ; \mathrm{SE}=0.015$, and number of phasic peaks: $M=8.68, \mathrm{SE}=1.36$ ). No interaction effects between noise and breakfast on mean phasic peak amplitude and number of phasic peaks were found.

Regarding EEG, breakfast did not affect the EEG alpha power, nor the EEG theta power. No effects of noise on alpha power or theta power were found. Also no interaction effects of breakfast and noise on alpha power or theta power were found.

No effect of breakfast was found on the P300 amplitude for target letters or non-target letters. Noise did affect P300 amplitude for target letters, $F(1,19)=49.31, p<0.001$, and non-target letters $(F(1,19)=71.63, p<0.001$. Noise bursts increased the P300 amplitude for both target $(M=5.34$; $\mathrm{SE}=0.47)$ and non-target letters $(M=4.06 ; \mathrm{SE}=0.35)$ compared to no-noise bursts (target letters: $M=3.81$; $\mathrm{SE}=0.40$, non-target letters: $M=3.14 ; \mathrm{SE}=0.29)$. Note that only letters without concurrent noise burst were taken into account-thus, effects of noise do not reflect a direct response to the noise burst itself, but the embedding of the letter in a noisy context. No interaction effects of breakfast and noise on P300 amplitude for target letters or non-target letters were found.

Additional repeated-measures ANOVAs were done to analyze the effect of type of letter in the 2-back task and noise, on the EEG P300 amplitude. Noise significantly affected P300 response to letters, $F(1,19)=65.24, p<0.001$, with higher P300 amplitude for noise bursts compared to nonoise bursts. A significant effect of type of letter was found on the EEG P300 amplitude, $F(1,19)=22.67, p<0.001$. As commonly found, the P300 amplitude was higher for target than non-target letters. Also an interaction effect of type of letters and noise on P300 amplitude was found, $F(1,19)=13.67, p=0.002$. Noise bursts increased the differentiation between target and non-target letters, indicating well-directed attention. Figure 2 shows the significant effect of this analysis.

An overview of the (close to) significant and non-significant results of stressors is shown in Table 3.

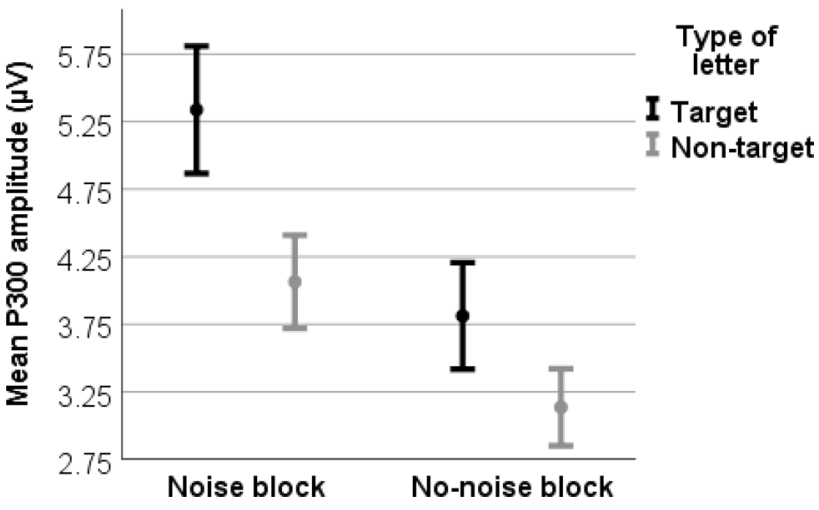

Fig. 2 Significant main and interaction effects of type of letters (target vs. non-target) and noise on P300 amplitude. Error bars are $\pm 1 \mathrm{SE}$ (standard error)

\section{Discussion}

This study examined potential interactions between two different stressors on cognitive performance: a metabolic stressor (skipping breakfast) and a sensory stressor (exposure to noise bursts). The experiment was designed to enable the comparison of the effect of the combined stressors with the effect of each individual stressor. The results did not confirm an interaction between the two stressors on the cognitive tasks. When both stressors were more effective in changing cognitive performance than either stressor alone through (dis)similar mechanisms, interaction effects would have been expected (Lloyd and Havenith 2016). Note that we cannot conclude that no interaction effects between noise bursts and skipping a meal exist at all, because it may have been the case that the effect of one of our stressors on cognitive performance was too small, which may have prevented an effect of the combination of stressors. Yet, it is conceivable that even if one of the two stressors that has no effect individually, it would have had an effect in combination with another subtle stressor. Although the stressors used in this study did not seem stressful enough to strongly interfere with cognitive performance, the effects of the individual stressors can help to understand in more detail why no interactions effects were found either.

The manipulation check, based on level of blood glucose, indicated that skipping breakfast did not lead to hypoglycemia. However, the subjective ratings showed that the participants experienced a greater feeling of hunger. An effect of skipping breakfast was also found on physiological variables that are indicative of a lower state of arousal after skipping breakfast (i.e., lower heart rate and lower EDA) compared to after consuming a standardized breakfast. This is in line with studies from Pivik (2006) and Nose et al. (2011) who found lower heart rates, indicating higher parasympathetic activity, after breakfast skipping. The cognitive tasks may 
not be sensitive enough to clearly detect a decline, while the physiological measures are more sensitive. It is plausible that, rather than a lack of blood glucose, this lower state of arousal and/or the subjective discomfort underly the trend for more misses on the 2-back task.

A suggestion for future research is to prolong the period of fasting, such that hypoglycemic conditions are reached. Our study design only used fasting from $8 \mathrm{pm}$ the night before the test day, while it appeared that the body was able to compensate for this lack of blood glucose. Prolonged fasting may result in stronger changes in cognitive performance and clearer interaction effects. The exercise was included to further lower blood glucose levels and thereby strengthening the effects of skipping breakfast. However, exercise can also increase arousal and enhance cognitive task performance (De Sousa et al. 2019; Lambourne and Tomporowski 2010), reducing rather than enhancing the effect of skipping breakfast. However, note that exercise was performed on both test days, and any increase (rather than decrease) of performance would have occurred in both conditions. The same applies for withdrawal from caffeine (tea or coffee). Participants were not allowed to drink coffee or tea, because the breakfast was standardized. Caffeine withdrawal in the morning may lower mental alertness and/or affect other cognitive functions negatively (Rogers et al 2013). However, as we have incorporated this in the protocol on both test day, any decrease would have occurred in both conditions.

The effect of noise bursts differed for the different dependent variables. Although the reaction time and accuracy on the 2-back task were not affected, noise bursts did lead to less missed responses, which is indicative of improved performance. It was not the case that more false alarms were found during the noise condition, implying that participants did not simply press the button more often. In contrast to the 2-back task, noise bursts reduced performance on the delayed recall test, evidenced by less memorized items. The physiological measures showed a higher state of arousal and vigilant attention under conditions of noise. The higher state of arousal suggests more invested effort for noise compared to no-noise. Noise conditions were associated with higher EDA responses, as EDA increases with a higher state of arousal (Critchley 2002). A state of increased attention during noise conditions was suggested by a higher (target-) P300 amplitude (Wild-Wall et al. 2011; Evans et al. 2011). Although not the interaction we were looking for, a combined effect of being more aroused under conditions of noise and the presence of a target letter on the p300 appears to be similar to an additive interaction effect. Besides this, alpha power showed a non-significant trend of lower activity during noise conditions, which would also be consistent with higher effort. This more aroused and attentive state was also experienced as higher load and stress based on the subjective ratings. Combining these results can indicate that participants invested additional effort to maintain a constant working memory performance level. The results may explain the lack of effects of stressors on cognitive performance as determined by 2-back reaction time and number of correct responses. The compensatory increase of mental effort has been supported with reported measures and markers of effort before (Szalma and Hancock 2011).

The effects of noise bursts on task performance in combination with the physiological and subjective workload measures are interesting in relation to an existing model about the relationship between task demands, performance, workload and effort (Veltman et al. 2003). This relationship is schematically depicted in Fig. 3a. Veltman et al. (2003) observed an inverted-U relation between stress or arousal and performance in a complex task situation. They added mental effort to the original inverted- $U$ curve from Yerkes and Dodson (1908), and labeled the $x$-axis as difficulty of the task (level of task demands) rather than stress or arousal. Four situations can be explained with a

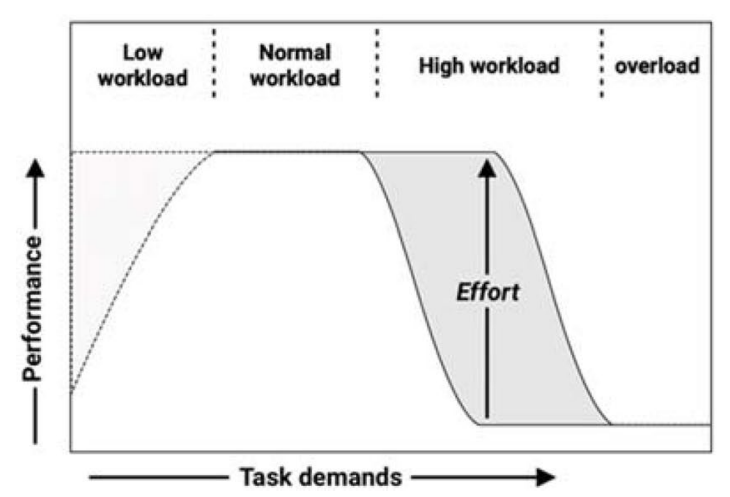

b

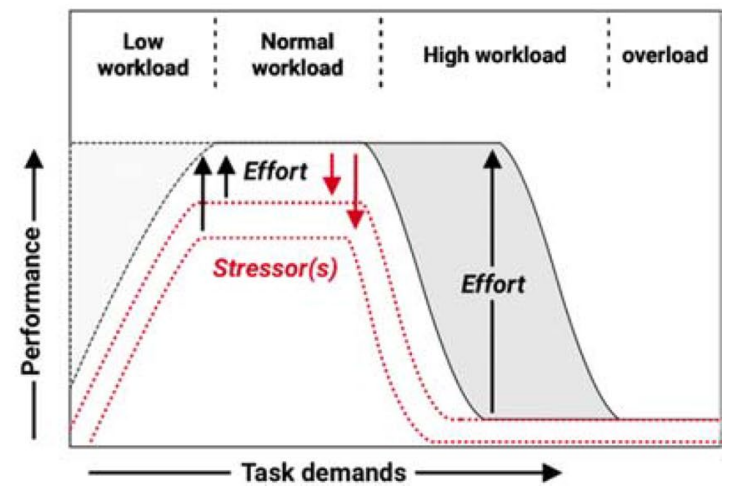

Fig. 3 Hypothetical relation between task demands, performance, workload, and effort (obtained from Veltman et al. 2003). a Original model; b Adaptation to the model 
this model: low workload with low performance due to an inattentive state, normal load with optimal performance, high workload with optimal performance through investment of additional effort, and overload where there are no (attentional) resources available, and it is no longer possible to invest additional effort. Performance, subjective, and physiological measures can be used to distinguishing between these four situations.

However, rather than that this means we are measuring at the right end of the $x$-axis in Fig. 3a (the part under high workload), the finding of less missed responses on the 2-back task with noise bursts can imply we are measuring at the part under normal workload of the $x$-axis. Although the task was in fact not easy, participants may have been in this far left end of the graph in conditions without noise because of an inattentive state. This suggests that noise bursts may help to keep participants alert, though the same noise can be disrupting in other types of cognitive performance, as we have shown for the effect of noise on the delayed recall of the verbal list learning task in line with a study from Cassidy and MacDonald (2007). An explanation for this more alerting state and improved performance (less missed responses on the 2-back task) when the letter coincides with a noise burst compared to the situation in which the letter was presented without a noise burst is based on multisensory integration (Van der Burg et al. 2008; Sumby and Pollack 1954; McGurk and MacDonald 1976). Indeed, it might be feasible that the presentation of the noise burst had a significant effect on the percept of the visual letter, as it is known that a visual event is perceived as more salient when it coincides with an auditory signal than when it is presented in isolation (see Van der Burg et al. 2011). Whereas Van der Burg and colleagues illustrated that a sound improves visual performance in a spatial context (i.e., a visual search task whereby search times were affected by the presentation of a sound), others reported comparable improvements in terms of accuracy (like in the present study) using a visual temporal task (Vroomen and de Gelder 2000; Olivers and Van der Burg 2008). An alternative explanation for the improved performance is based on temporal preparation. From the timing literature, we know that if participants receive a warning signal before a visual target, then participants respond faster to the target when the time between the temporal warning signal is large than when it is small (Los and Van der Burg 2013). In our study, the noise burst, if present, always coincided with the visual letter. However, this does not mean that participants were unable to prepare for the upcoming target. Indeed, it is known from the literature that auditory information is faster processed in the brain than visual information (Jain et al. 2015), making it feasible for participants to prepare for the upcoming visual target when it coincides with an auditory signal (Los and
Van der Burg 2013). In our study, this implies that noise bursts make it feasible for the participants to prepare for the upcoming letter in the 2-back task, explaining why performance was better when the letter coincides with a noise burst compared to the condition without noise bursts. Whether the more alerting state in this study is a result of multisensory integration or temporal preparation is not clear and more research is necessary to examine this.

Reflecting on the model proposed by Veltman et al. (2003), it is assumed that task demands include both the intrinsic difficulty of the task and the indirect impact of stressor(s). For example, it is possible that high effort on the left end of the model is necessary to maintain performance high at risk of inattentiveness, while this can occur separately from the ease of a task. Therefore, we argue for separating task difficulty and impact of stressors. This can be implemented by adding a new dimension 'stressors' to the model. This adjustment would allow us in future research to explicitly make the difference between the effect of stressor(s) and level of task difficulty to better understand and predict their (interactive) impact on performance. Accordingly, each individual stressor and their combined effect can be represented as separate lines in the model (see Fig. 3b). With the use of this model, we can better predict the (interactive) impact of stressors on cognitive performance in challenging environments.

In the end, this kind of research can be applied to get insight into mechanisms underlying the stressors, which can lead to better countermeasures that prevent or alleviate negative effects of combined stressors on performance (Martin et al. 2020). Taking a comprehensive approach of performance, physiological and subjective measures to study the impact of stressors on cognition might be valuable in strengthening industrial safety. For example, Vanderhaegen et al. (2020) discussed examples of supportive systems that can alert operators when they get tired. This is crucial in environments where different professionals have to maintain high performance.

\subsection{Conclusions}

In conclusion, this study found no interaction effect of a metabolic stressor (skipping breakfast) and a sensory stressor (exposure to noise bursts) on cognitive performance. The results of the individual stressors can be used for explaining the lack of an interaction. Skipping breakfast appeared to be ineffective to cause a hypoglycemic state, indicating that it was not an effective metabolic stressor. The lower state of arousal and subjective discomfort after breakfast skipping may explain the trend of more missed responses. Noise bursts appeared to generate arousal and overall increased attention. The physiological and subjective variables may 
be useful to reveal extra effort that people put into a task to maintain performance. Since the inverted U-shape model for workload does not take effect of environmental stressors into account, we propose an amendment to this model to incorporate such effects.

Acknowledgements The authors want to thank Martin van Schaik and Hanna Heyer for their contributions to the setup of the experiment. This work was funded by the Dutch Ministry of Defense (V1532 program) and the TNO research ERP-Body-Brain Interactions.

Author contributions All authors contributed to the study conception and design. Material preparation, data collection, and analysis were performed by $\mathrm{CB}$ and IS. The first draft of the manuscript was written by $\mathrm{CB}$ and all authors commented on previous versions of the manuscript. All authors read and approved the final manuscript.

Funding No external funding applicable.

Data availability The data that support the findings of this study are available on request from the corresponding author.

Code availability Not applicable.

\section{Compliance with ethical standards}

Conflict of interest The authors declare that they have no conflict of interest.

Ethical approval An approval for this study was granted by the TNO Internal Review Board (reference number: 2019-011).

Informed consent All participants involved in this study signed informed consent. The article does not indicate any participants by name.

Consent for publication This paper has not been published elsewhere in any form, and is currently not under consideration for publication elsewhere. All authors have seen and agreed to the submitted version of the paper.

Open Access This article is licensed under a Creative Commons Attribution 4.0 International License, which permits use, sharing, adaptation, distribution and reproduction in any medium or format, as long as you give appropriate credit to the original author(s) and the source, provide a link to the Creative Commons licence, and indicate if changes were made. The images or other third party material in this article are included in the article's Creative Commons licence, unless indicated otherwise in a credit line to the material. If material is not included in the article's Creative Commons licence and your intended use is not permitted by statutory regulation or exceeds the permitted use, you will need to obtain permission directly from the copyright holder. To view a copy of this licence, visit http://creativecommons.org/licenses/by/4.0/.

\section{References}

Adolphus K, Lawton CL, Champ CL, Dye L (2016) The effects of breakfast and breakfast composition on cognition in children and adolescents: a systematic review. Adv Nutr 7(3):590S-612S. https ://doi.org/10.3945/an.115.010256

Ayaz H, Izzetoglu M, Bunce S, Heiman-Patterson T, Onaral B (2007) Detecting cognitive activity related hemodynamic signal for brain computer interface using functional near infrared spectroscopy. In 2007 3rd International IEEE/EMBS Conference on Neural Engineering, pp. 342-345

Bell AJ, Sejnowski TJ (1995) An information-maximization approach to blind separation and blind deconvolution. Neural Comput 7(6):1129-1159. https://doi.org/10.1162/neco.1995.7.6.1129

Benedek M, Kaernbach C (2010) A continuous measure of phasic electrodermal activity. J Neurosci Methods 190(1):80-91. https://doi. org/10.1016/j.jneumeth.2010.04.028

Benton D, Parker PY (1998) Breakfast, blood glucose, and cognition. Am J Clin Nutr 67(4):772S-778S. https://doi.org/10.1093/ ajcn/67.4.772S

Brouwer AM, Hogervorst MA, Herman P, Kooi F (2009) Are you really looking? Finding the answer through fixation patterns and EEG. International conference on foundations of augmented cognition. Springer, Berlin, pp 329-338

Brouwer AM, Hogervorst MA, Van Erp JB, Heffelaar T, Zimmerman PH, Oostenveld R (2012) Estimating workload using EEG spectral power and ERPs in the n-back task. J Neural Eng 9(4):045008. https://doi.org/10.1088/1741-2560/9/4/045008

Brouwer AM, Hogervorst MA, Holewijn M, van Erp JB (2014) Evidence for effects of task difficulty but not learning on neurophysiological variables associated with effort. Int J Psychophysiol 93(2):242-252. https://doi.org/10.1016/j.ijpsy cho.2014.05.004

Cassidy G, MacDonald RA (2007) The effect of background music and background noise on the task performance of introverts and extraverts. Psychol Music 35(3):517-537. https://doi. org/10.1177/0305735607076444

Critchley HD (2002) Electrodermal responses: what happens in the brain. Neuroscientist 8(2):132-142. https://doi. org/10.1177/107385840200800209

Critchley HD, Garfinkel SN (2018) The influence of physiological signals on cognition. Curr Opin Behav Sci 19:13-18. https:// doi.org/10.1016/j.cobeha.2017.08.014

De Sousa AFM, Medeiros AR, Del Rosso S, Stults-Kolehmainen M, Boullosa DA (2019) The influence of exercise and physical fitness status on attention: a systematic review. Int Rev Sport Exerc Psychol 12(1):202-234. https://doi.org/10.1080/17509 84X.2018.1455889

Delorme A, Makeig S (2004) EEGLAB: an open source toolbox for analysis of single-trial EEG dynamics including independent component analysis. J Neurosci Methods 134(1):9-21. https:// doi.org/10.1016/j.jneumeth.2003.10.009

Evans DE, Craig C, Oliver JA, Drobes DJ (2011) The smoking N-back: a measure of biased cue processing at varying levels of cognitive load. Nicotine Tob Res 13(2):88-93. https://doi.org/10.1093/ntr/ ntq 214

Fink A, Grabner RH, Neuper C, Neubauer AC (2005) EEG alpha band dissociation with increasing task demands. Cogn Brain Res 24(2):252-259. https://doi.org/10.1016/j.cogbrainres.2005.02.002

Galioto R, Spitznagel MB (2016) The effects of breakfast and breakfast composition on cognition in adults. Adv Nutr 7(3):576S-589S. https://doi.org/10.3945/an.115.010231

Hockey GRJ (1986) Changes in operator efficiency as a function of environmental stress, fatigue, and circadian rhythms. In: Boff KR, Kaufman L, Thomas JP (eds) Handbook of perception and human performance, vol 2. Wiley, New York, pp 1-49

Hoyland A, Dye L, Lawton CL (2009) A systematic review of the effect of breakfast on the cognitive performance of children and adolescents. Nutr Res Rev 22(2):220-243. https://doi.org/10.1017/ S0954422409990175 
Izzetoglu M, Bunce SC, Izzetoglu K, Onaral B, Pourrezaei K (2007) Functional brain imaging using near-infrared technology. IEEE Eng Med Biol Mag 26(4):38-46. https://doi.org/10.1109/ MEMB.2007.384094

Jain A, Bansal R, Kumar A, Singh KD (2015) A comparative study of visual and auditory reaction times on the basis of gender and physical activity levels of medical first year students. Int J Appl Basic Med Res 5(2):124. https://doi.org/10.4103/2229-516X.157168

Jann K, Dierks T, Boesch C, Kottlow M, Strik W, Koenig T (2009) BOLD correlates of EEG alpha phase-locking and the fMRI default mode network. Neuroimage 45(3):903-916. https://doi. org/10.1016/j.neuroimage.2009.01.001

Jasper HH (1958) The ten-twenty electrode system of the International Federation. Electroencephalogr Clin Neurophysiol 10:370-375. https://doi.org/10.1080/00029238.1961.11080571

Jensen O, Tesche CD (2002) Frontal theta activity in humans increases with memory load in a working memory task. Eur J Neurosc 15(8):1395-1399. https://doi.org/10.104 6/j.1460-9568.2002.01975.x

Kirchner WK (1958) Age differences in short-term retention of rapidly changing information. J Exp Psychol 55(4):352. https://doi. org $/ 10.1037 / \mathrm{h} 0043688$

Lambourne K, Tomporowski P (2010) The effect of exercise-induced arousal on cognitive task performance: a meta-regression analysis. Brain Res 1341:12-24. https://doi.org/10.1016/j.brain res.2010.03.091

Laufs H, Krakow K, Sterzer P et al (2003) Electroencephalographic signatures of attentional and cognitive default modes in spontaneous brain activity fluctuations at rest. Proc Natl Acad Sci 100(19):11053-11058. https://doi.org/10.1073/pnas.1831638100

Lieberman HR, Niro P, Tharion WJ et al (2006) Cognition during sustained operations: comparison of a laboratory simulation to field studies. Aviat Space Environ Med 77(9):929-935

Lloyd A, Havenith G (2016) Interactions in human performance: an individual and combined stressors approach. Temperature 3(4):514-517. https://doi.org/10.1080/23328940.2016.1189991

Lloyd A, Hodder S, Havenith G (2015) The interactive effect of cooling and hypoxia on forearm fatigue development. Eur J Appl Physiol 115(9):2007-2018. https://doi.org/10.1007/s00421-015-3181-1

Lloyd A, Raccuglia M, Hodder S, Havenith G (2016) Interaction between environmental temperature and hypoxia on central and peripheral fatigue during high-intensity dynamic knee extension. J Appl Physiol 120(6):567-579. https://doi.org/10.1152/japplphysi ol.00876.2015

Los SA, Van der Burg E (2013) Sound speeds vision through preparation, not integration. J Exp Psychol Hum Percept Perform 39(6):1612. https://doi.org/10.1037/a0032183

Martin K, McLeod E, Périard J, Rattray B, Keegan R, Pyne DB (2019) The impact of environmental stress on cognitive performance: a systematic review. Hum Factors 61(8):1205-1246. https://doi. org/10.1177/0018720819839817

Martin K, Périard J, Rattray B, Pyne DB (2020) Physiological factors which influence cognitive performance in military personnel. Hum Factors 62(1):93-123. https://doi.org/10.1177/0018720819 841757

Matthews G, Davies DR, Stammers RB, Westerman SJ (2000) Human performance: cognition, stress, and individual differences. Psychology Press, New York

McGurk H, MacDonald J (1976) Hearing lips and seeing voices. Nature 264(5588):746-748. https://doi.org/10.1038/264746a0

Mendes WB (2009) Assessing autonomic nervous system activity. In: Harmon-Jones E, Beer JS (eds) Methods in social neuroscience. Guilford Press, New York, pp 118-147

Mulder LJM, Mulder G (1987) Cardiovascular reactivity and mental workload. In: Kitney RI, Rompelman O (eds) The beat-by-beat investigation of cardiovascular function. Clarendon Press, Oxford, pp 216-253

Nose $Y$ et al (2011) Effects of eating or skipping breakfast on heart rate and oxygen uptake. Kawasaki Med Welf J 16(2):58-63. https ://doi.org/10.15112/00005029

Olivers CN, Van der Burg E (2008) Bleeping you out of the blink: sound saves vision from oblivion. Brain Res 1242:191-199. https ://doi.org/10.1016/j.brainres.2008.01.070

Pan J, Tompkins WJ (1985) A real-time QRS detection algorithm. IEEE Trans Biomed Eng 32(3):230-236. https://doi.org/10.1109/ TBME.1985.325532

Paulus MP, Potterat EG, Taylor MK et al (2009) A neuroscience approach to optimizing brain resources for human performance in extreme environments. Neurosci Biobehav Rev 33(7):1080-1088. https://doi.org/10.1016/j.neubiorev.2009.05.003

Pfurtscheller G, Stancak A Jr, Neuper C (1996) Event-related synchronization (ERS) in the alpha band-an electrophysiological correlate of cortical idling: a review. Int J Psychophysiol 24(1-2):39-46. https://doi.org/10.1016/S0167-8760(96)00066-9

Pivik RT, Dykman RA, Tennal K, Gu Y (2006) Skipping breakfast: gender effects on resting heart rate measures in preadolescents. Physiol Behav 89(2):270-280. https://doi.org/10.1016/j.physb eh.2006.06.001

Polich J (2007) Updating P300: an integrative theory of P3a and P3b. Clin Neurophysio 118 (10):2128-02148. https://doi.org/10.1016/j. clinph.2007.04.019

Rogers PJ, Heatherley SV, Mullings EL, Smith JE (2013) Faster but not smarter: effects of caffeine and caffeine withdrawal on alertness and performance. Psychopharmacology 226(2):229-240. https:// doi.org/10.1007/s00213-012-2889-4

Sumby WH, Pollack I (1954) Visual contribution to speech intelligibility in noise. J Acoust Soc Am 26(2):212-215. https://doi. org/10.1121/1.1907309

Szalma JL, Hancock PA (2011) Noise effects on human performance: a meta-analytic synthesis. Psychol Bull 137(4):682

Thompson TA, Wilson PH, Snyder PJ et al (2011) Sensitivity and testretest reliability of the international shopping list test in assessing verbal learning and memory in mild Alzheimer's disease. Arch Clin Neuropsychol 26(5):412-424. https://doi.org/10.1093/arcli $\mathrm{n} / \mathrm{acr} 039$

Tipton M (2012) A case for combined environmental stressor studies. Extreme Physiol Med 1(1):7. https://doi. org/10.1186/2046-7648-1-7

Van der Burg E, Olivers CN, Bronkhorst AW, Theeuwes J (2008) Pip and pop: nonspatial auditory signals improve spatial visual search. J Exp Psychol Hum Percept Perform 34(5):1053. https:// doi.org/10.1037/0096-1523.34.5.1053

Van der Burg E, Talsma D, Olivers CN, Hickey C, Theeuwes J (2011) Early multisensory interactions affect the competition among multiple visual objects. Neuroimage 55(3):1208-1218. https:// doi.org/10.1016/j.neuroimage.2010.12.068

Vanderhaegen F, Wolff M, Mollard R (2020) Non-conscious errors in the control of dynamic events synchronized with heartbeats: a new challenge for human reliability study. Saf Sci 129:104814. https:// doi.org/10.1016/j.ssci.2020.104814

Veltman JA, Jansen C, Hockey GRJ, Gaillard AWK, Burov O (2003) Differentiation of mental effort measures: consequences for adaptive automation. NATO science series I: life and behavioural sciences. ISO press, Amsterdam, pp 249-259

Vroomen J, Gelder BD (2000) Sound enhances visual perception: cross-modal effects of auditory organization on vision. J Exp Psychol Hum Percept Perform 26(5):1583. https://doi. org/10.1037/0096-1523.26.5.1583

Wild-Wall N, Falkenstein M, Gajewski PD (2011) Age-related differences in working memory performance in a 2-back task. Front Psychol 2:186. https://doi.org/10.3389/fpsyg.2011.00186 
Winkler I, Haufe S, Tangermann M (2011) Automatic classification of artifactual ICA-components for artifact removal in EEG signals. Behav Brain Funct 7(1):30. https://doi. org/10.1186/1744-9081-7-30

Yerkes RM, Dodson JD (1908) The relation of strength of stimulus to rapidity of habit-formation. J Comp Neurol Psychol 18(5):459482. https://doi.org/10.1002/cne.920180503

Yong JSE, Wang DY (2015) Impact of noise on hearing in the military. Mil Med Res 2(1):6. https://doi.org/10.1186/s40779-015-0034-5
Zijlstra FRH, Van Doorn L (1985) The construction of a scale to measure subjective effort. Delft (Netherlands) 43:124-139

Publisher's Note Springer Nature remains neutral with regard to jurisdictional claims in published maps and institutional affiliations. 